\title{
Efficacy of Abatacept for Arthritis in Patients with an Overlap Syndrome between Rheumatoid Arthritis and Systemic Lupus Erythematosus
}

\author{
Kei Ikeda, Yoshie Sanayama, Sohei Makita, Junichi Hosokawa, Mieko Yamagata, \\ Daiki Nakagomi, Katsuhiko Takabayashi, and Hiroshi Nakajima
}

\begin{abstract}
Department of Allergy and Clinical Immunology, Chiba University Hospital, 1-8-1 Inohana, Chuo-ku, Chiba-shi, Chiba 260-8670, Japan
\end{abstract}

Correspondence should be addressed to Kei Ikeda; k.ikeda@faculty.chiba-u.jp

Received 1 September 2013; Accepted 1 October 2013

Academic Editor: T. Nakayama

Copyright (C) 2013 Kei Ikeda et al. This is an open access article distributed under the Creative Commons Attribution License, which permits unrestricted use, distribution, and reproduction in any medium, provided the original work is properly cited.

\begin{abstract}
Introduction. This study aimed to investigate the efficacy of abatacept for arthritis in patients with rhupus, an overlap syndrome between rheumatoid arthritis (RA) and systemic lupus erythematosus (SLE). Methods. Patients who fulfilled both the 2010 ACR/EULAR criteria for RA classification and the 1997 ACR revised criteria for classification of SLE and received abatacept treatment for arthritis were retrospectively studied. Results. Six rhupus patients who fulfilled the inclusion criteria above were identified. All patients had active arthritis despite receiving antirheumatic drugs including methotrexate when abatacept was initiated. Clinical Disease Activity Index (CDAI) significantly decreased between baseline and 12 weeks $(P=0.028)$ and remained low through 24 weeks. All patients achieved either a good or moderate response according to the EULAR response criteria at 24 weeks. Health Assessment Questionnaire-Disability Index (HAQ-DI) also significantly decreased between baseline and 24 weeks $(P=0.043)$. In addition, the levels of immunoglobulin $\mathrm{G}$ and anti-DNA antibody significantly decreased between baseline and 24 weeks ( $P=0.028$ and $P=0.043$, resp.). Conclusions. Treatment with abatacept is likely to be efficacious in patients with rhupus whose arthritis is refractory to methotrexate. In addition, abatacept may have a moderate effect on abnormal antibody production in rhupus patients.
\end{abstract}

\section{Introduction}

The clinical coexistence of rheumatoid arthritis (RA) and systemic lupus erythematosus (SLE) is a rare occurrence frequently referred to as "rhupus syndrome" [1]. Increasing evidence suggests that arthritis in patients with rhupus can cause joint damage indistinguishable from that of RA, requiring aggressive treatment [2-5]. However, TNF antagonists, which are the most potent agents in preventing joint damage in RA when used in combination with methotrexate (MTX), can induce production of autoantibodies characteristic to SLE such as antinuclear antibodies (ANA) or anti-DNA antibodies [6,7]. Less frequently but more importantly, TNF antagonists can cause lupus manifestations in RA [6-10] and rhupus syndrome [11].

Abatacept is a fully human, soluble fusion protein that consists of the extracellular domain of human cytotoxic
T-lymphocyte antigen 4 (CTLA-4) and the Fc portion of IgG1, which selectively modulates the CD80/CD86:CD28 costimulatory signals and interactions between activated $\mathrm{T}$ cells and antigen presenting cells (APCs). The use of abatacept in patients with RA is associated with sustained efficacy both in disease activity and in radiographic progression without inducing autoantibody production [12-16]. Abatacept treatment has been explored for its efficacy in other T cell-mediated diseases such as ankylosing spondylitis $[17,18]$. Moreover, a recent phase IIb randomized, doubleblind, placebo-controlled trial showed modest but significant efficacy of abatacept against polyarthritis in patients with non-life-threatening SLE [19]. However, abatacept treatment in rhupus patients has not been reported.

In this study, we retrospectively assessed the efficacy of abatacept in six rhupus patients with active arthritis but not with life-threatening lupus manifestations. 

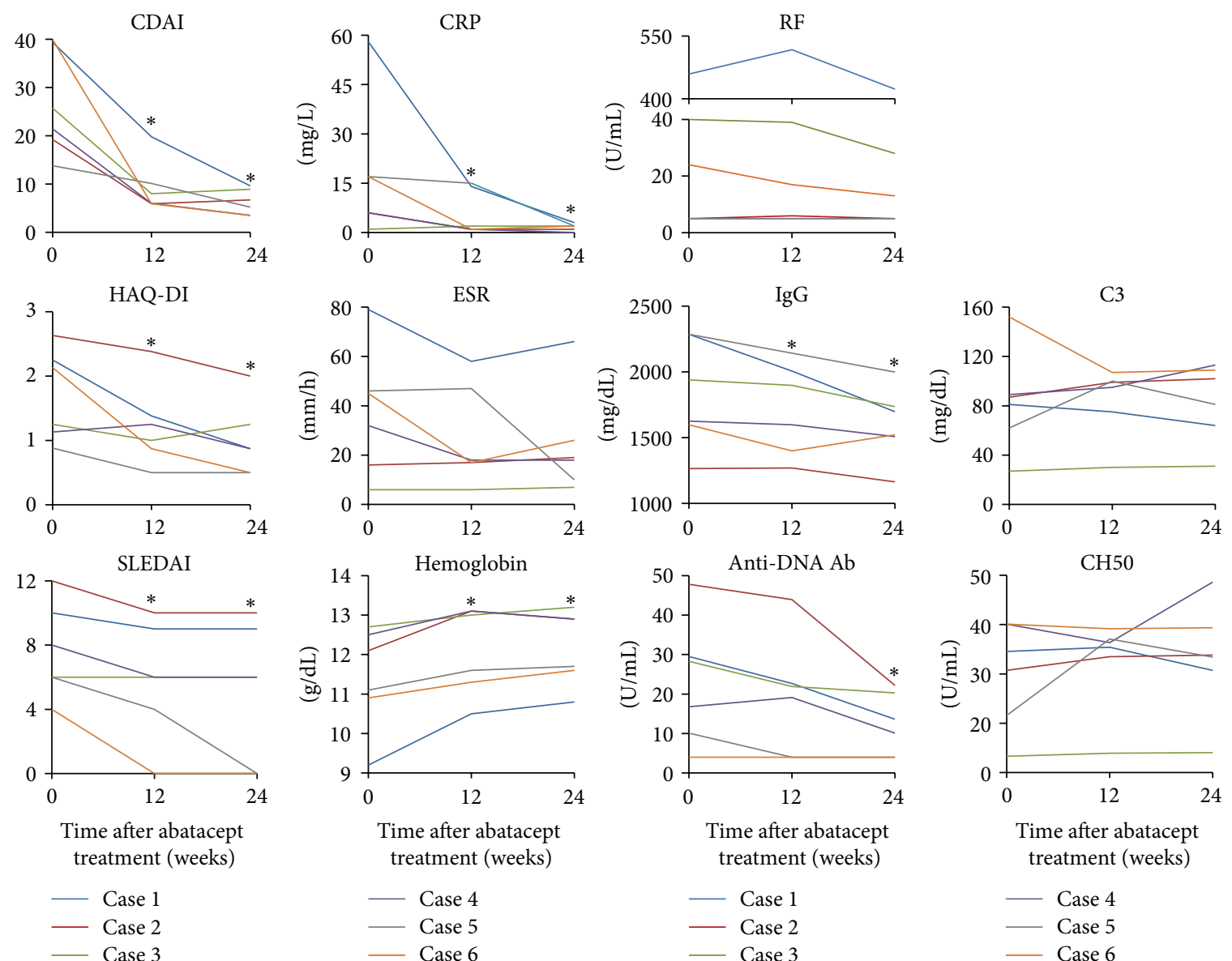

FIGURE 1: Changes in clinical indices and laboratory tests reflecting disease activity of RA and/or SLE in each case during 24 weeks after abatacept treatment. ${ }^{*} P<0.05$, Wilcoxon's signed-rank test. Comparisons were made against baseline values. RA: rheumatoid arthritis; SLE: systemic lupus erythematosus; CDAI: Clinical Disease Activity Index; HAQ-DI: Health Assessment Questionnaire-Disability Index; SLEDAI: systemic lupus erythematosus disease activity index; CRP: C-reactive protein; ESR: erythrocyte sedimentation rate; RF, rheumatoid factor; IgG: immunoglobulin G; Anti-DNA Ab: anti-DNA antibody.

\section{Materials and Methods}

2.1. Patients. Medical records in the Department of Allergy and Clinical Immunology, Chiba University Hospital were thoroughly reviewed to identify patients who received abatacept treatment for arthritis and also fulfilled both the 2010 ACR/EULAR criteria for RA classification and the 1997 ACR revised criteria for classification of SLE. In order to ensure the inclusion of patients with genuine overlap, patients were excluded when the arthritis was better explained by SLE than by RA, and arthritis was not counted when SLE was classified. All patients gave a written consent for their clinical information to be published and the study procedures were approved by the Ethics Committee of Chiba University.

2.2. Statistical Analysis. Statistical analysis was performed using SPSS version 16.0J (IBM Japan, Tokyo, Japan). As all data were not normally distributed, data were summarized with medians and were analyzed using nonparametric tests
(Wilcoxon's signed-rank test). $P$ values less than 0.05 were considered significant.

\section{Results}

3.1. Demographics and Disease Characteristics of RA. Six patients who fulfilled the above mentioned inclusion criteria were identified. Demographics and disease characteristics of RA before abatacept administration of these patients are summarized in Table 1. All patients were Japanese females with a median age of 57.5 years. Four patients had an onset of arthritis symptoms which preceded the diagnosis of SLE. Three patients were seronegative (i.e., both rheumatoid factor $[\mathrm{RF}]$ and anticitrullinated protein antibody [ACPA] were negative) at baseline although one of them (Case 5) was positive for RF at the time of the diagnosis of SLE. Five patients had at least one erosive lesion on radiograph that was typical of RA. Median level of C-reactive protein (CRP) at baseline was relatively low $(11.5 \mathrm{mg} / \mathrm{L})$ as compared to median Clinical Disease Activity Index (CDAI) (23.55) (Figure 1). 


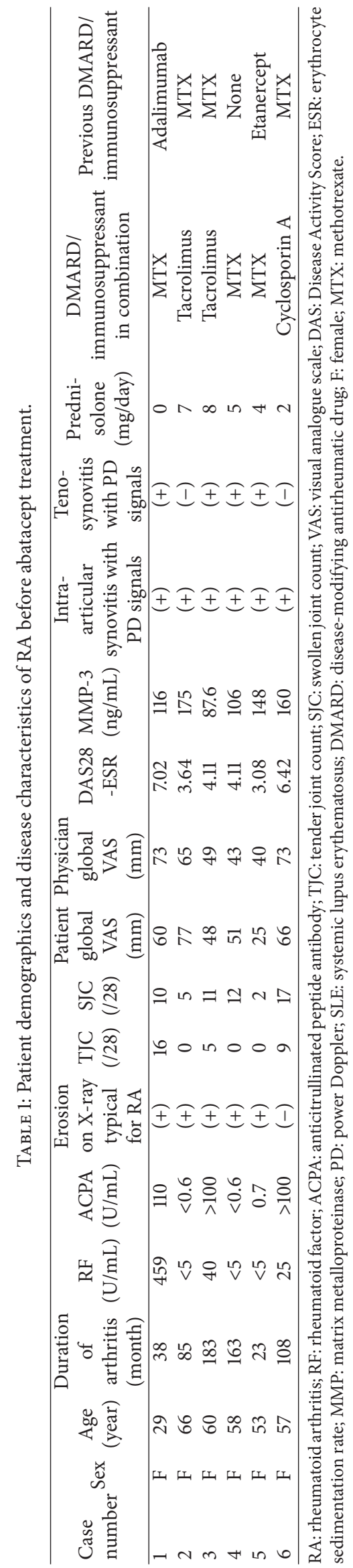


All patients underwent musculoskeletal ultrasonography for the assessment of synovial inflammation before abatacept treatment. All patients had increased PD signals within intraarticular synovium (i.e., active intra-articular synovitis) in at least one joint region, and five out of six patients had increased PD signals within tenosynovium as well (i.e., active tenosynovitis) in at least one joint region.

3.2. Disease Characteristics of SLE. Disease characteristics of SLE before abatacept treatment are summarized in Table 2. No patient had previously experienced severe organ manifestations of SLE such as nephritis or neuropsychiatric lupus, and arthritis was the major disease manifestation when abatacept was introduced. Sjogren's syndrome was the most common concomitant autoimmune disease other than RA or SLE $(n=4)$, followed by chronic thyroiditis $(n=$ 2 ) and scleroderma $(n=1)$. All patients were positive for antinuclear antibody and five patients were positive for anti-DNA antibody at the baseline. Antidouble stranded DNA antibody in Case 6 was positive when the patient was diagnosed with SLE but turned negative under treatment. Other autoantibodies, which, were positive at baseline were anti-Ro antibody $(n=4)$, anti-U1-RNP antibody $(n=3)$, anti-La antibody $(n=2)$, anti-thyroid peroxidase antibody $(n=2)$, anti-cardiolipin antibody $(n=1)$, and lupus anticoagulant $(n=1)$. Reflecting the lack of severe organ manifestations, clinically significant hypocomplementemia was only present in one patient (Case 3).

3.3. Previous Treatment. Five patients were receiving a small dose of prednisolone (median $4.5 \mathrm{mg} /$ day), whereas one patient (Case 1) was not because the patient did not agree to receive corticosteroid therapy. All patients were receiving treatment with MTX although half of them $(n=3)$ discontinued MTX due to either cytopenia, elevation of liver enzymes, or lack of efficacy when abatacept therapy was initiated. Three patients were receiving a calcineurin inhibitor such as tacrolimus $(n=2)$ or cyclosporine A $(n=1)$, effectiveness of which was insufficient. Two patients had previously received a TNF antagonist, which was not effective in either case. One patient (Case 1) received adalimumab before the diagnosis of SLE. Although the patient had already tested positive for anti-nuclear antibody (ANA) and anti-DNA antibody before receiving adalimumab and it was discontinued after only four injections 7 months before the development of her major lupus symptoms; the TNF $\alpha$ blockade could have been the trigger for the onset of SLE in her case. No patient had received rituximab because the use of rituximab has been approved for neither RA nor SLE in Japan.

3.4. Efficacy of Abatacept for Arthritis. As shown in Figure 1, CDAI and CRP significantly decreased after 12 weeks of abatacept treatment (median CDAI 23.55 versus 7 and $P=0.028$; median CRP $11.5 \mathrm{mg} / \mathrm{L}$ versus $1.5 \mathrm{mg} / \mathrm{L}$ and $P=0.046)$ and remained low through 24 weeks (median CDAI 5.95; median CRP $2.0 \mathrm{mg} / \mathrm{L}$ ). Four patients achieved a good or moderate response according to EULAR response criteria at 12 weeks (three good and one moderate) and all patients achieved a good or moderate response at 24 weeks (two good and four moderate). Health Assessment Questionnaire-Disability Index (HAQ-DI) also significantly decreased from median of 2.13 at baseline to median of 0.87 at 12 weeks $(P=0.046)$ and median of 0.5 at 24 weeks $(P=0.043)$. In addition, matrix metalloproteinase 3 (MMP-3) also significantly decreased from median of $132 \mathrm{ng} / \mathrm{mL}$ at baseline to median of $88 \mathrm{ng} / \mathrm{mL}$ at 12 weeks $(P=0.028)$ and median of $76.7 \mathrm{ng} / \mathrm{mL}$ at 24 weeks $(P=0.028)$ (data not shown). Prednisolone dose was decreased slightly but successfully by $1 \mathrm{mg}$ /day in Case 3 and 6 before 24 weeks. These results suggest that abatacept is efficacious in the treatment of arthritis for patients with rhupus.

\subsection{Efficacy of Abatacept on Nonarticular Lupus Manifesta-} tions. As shown in Figure 1, Systemic Lupus Erythematosus Disease Activity Index (SLEDAI) significantly decreased from median of 7 at baseline to median of 6 at 12 and 24 weeks $(P=0.039$ and 0.042 , resp.), mostly reflecting the remission induction of arthritis in Cases 5 and 6 . Other major symptoms which, upon improvement, decreased SLEDAI were fever in Case 1 and rash in Case 5. Among laboratory tests for lupus activity, hemoglobin significantly increased from median of $11.6 \mathrm{~g} / \mathrm{dL}$ to median of $12.3 \mathrm{~g} / \mathrm{dL}$ at 12 and 24 weeks $(P=0.028)$. None of the six patients had thrombocytopenia and only one had leukocytopenia at baseline, which did not improve with abatacept treatment. The serum levels of immunoglobulin G (IgG) and anti-DNA antibody also significantly decreased from baseline to 24 weeks (IgG, median $1,782 \mathrm{mg} / \mathrm{dL}$ to $1,609.5 \mathrm{mg} / \mathrm{dL}$, and $P=0.028$; antiDNA antibody, median $22.55 \mathrm{U} / \mathrm{mL}$ to $11.85 \mathrm{U} / \mathrm{mL}$, and $P=$ 0.043). However, changes in $\mathrm{C} 3$ levels and $\mathrm{CH} 50$ were variable and were not statistically significant. These data suggest that the efficacy of abatacept on nonarticular lupus manifestation at low disease activity states may be absent but abatacept may have moderate effect on abnormal antibody production in rhupus patients.

3.6. Adverse Events. One patient (Case 5) experienced mild olecranon bursitis in the elbow after 2 nd administration of abatacept. The bursitis was considered to be self-limiting or infectious, since swelling in the other joints was improving and the symptom subsided after 1-week treatment with oral antibiotics. The 3rd administration was postponed for a week, but abatacept was restarted without relapse. No other adverse events, including exacerbation of any lupus manifestations or concomitant autoimmune conditions, have been reported for a median follow-up period of 15 months (range 7-22).

3.7. Case Report (Case 3). A 60-year-old woman was admitted to the Department of Allergy and Clinical Immunology, Chiba University Hospital, on 13th of November, 2009, for the treatment of RA and SLE. She had been diagnosed with RA since 1996, when she had arthritis in hands and tested positive for RF, but had only received Chinese herbal medicine since the diagnosis. She was diagnosed with duodenal cancer and SLE in August, 2009, when she was admitted to another hospital for the investigation of 


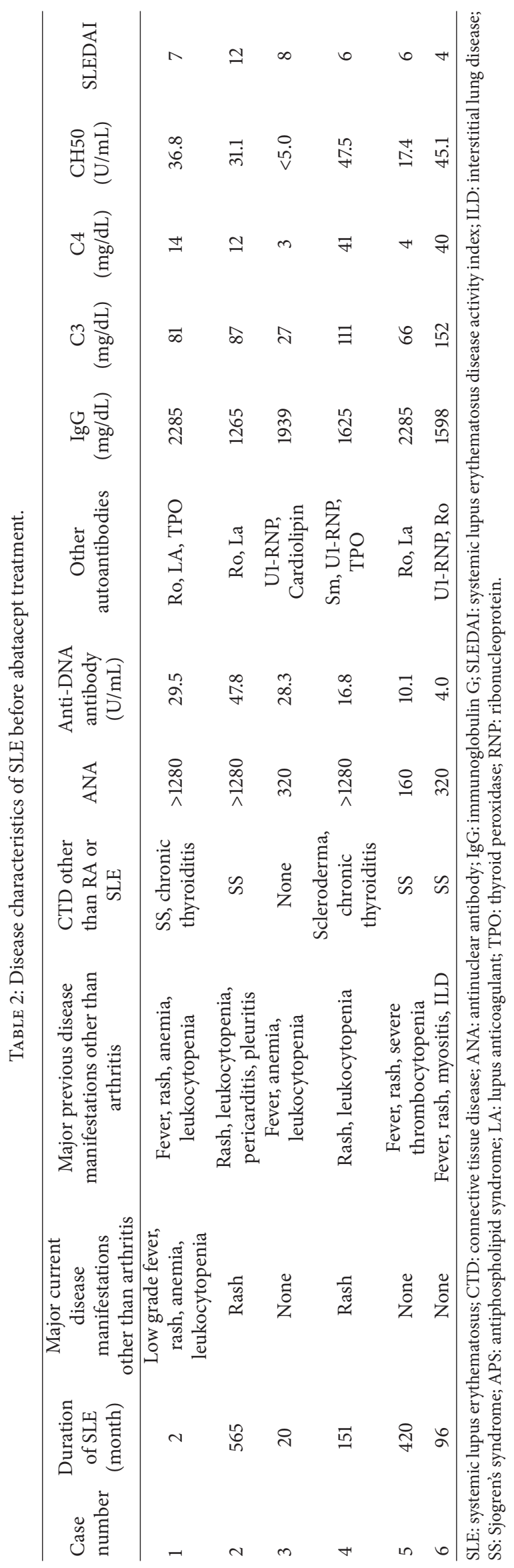




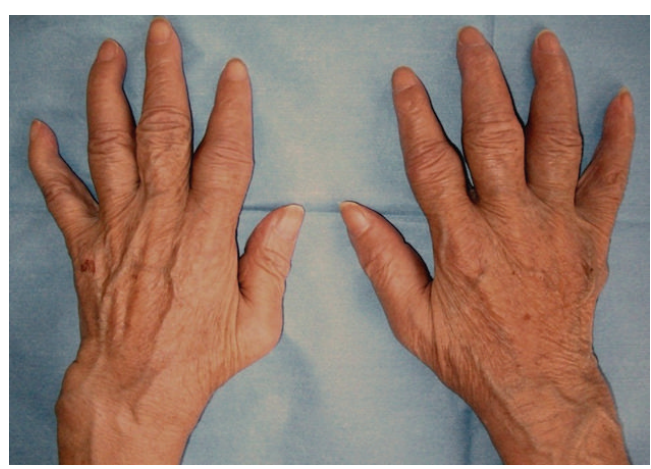

(a)

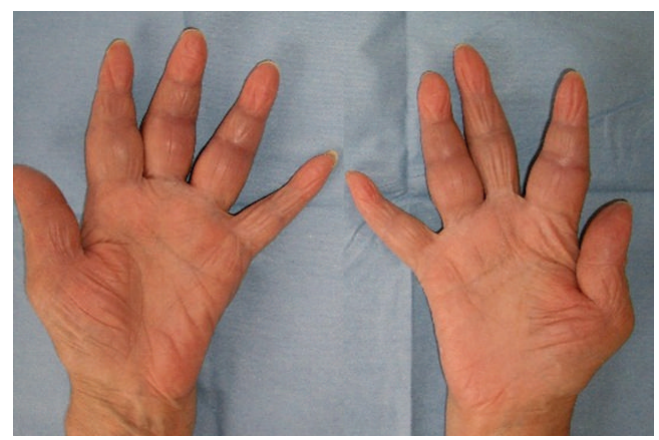

(b)

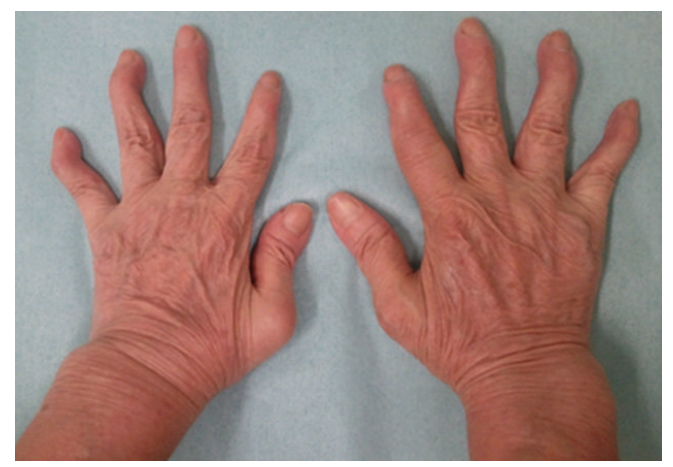

(c)

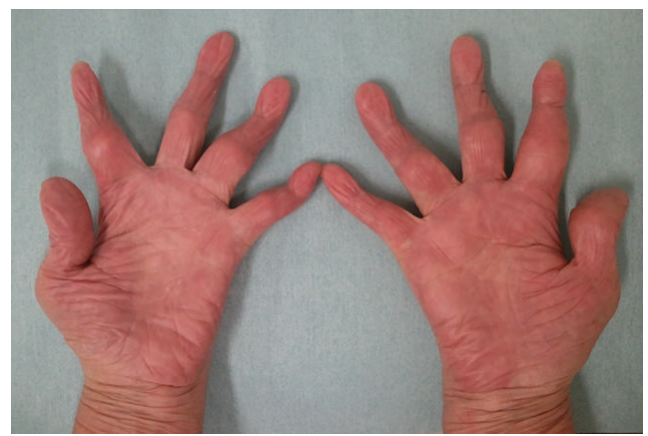

(d)

Figure 2: Joint swelling of hands in Case 3 before and after treatment. Dorsal aspects $(a, c)$ and palmer aspects (b, d) of the hand are shown. Swelling in fingers and wrists before treatment $(\mathrm{a}, \mathrm{b})$ markedly improved after 24 weeks of abatacept treatment $(c, d)$, leaving swan-neck deformity.

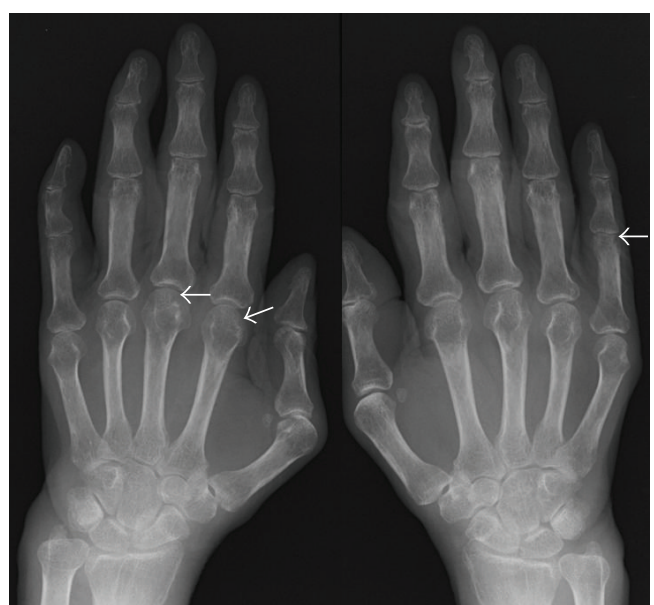

(a)

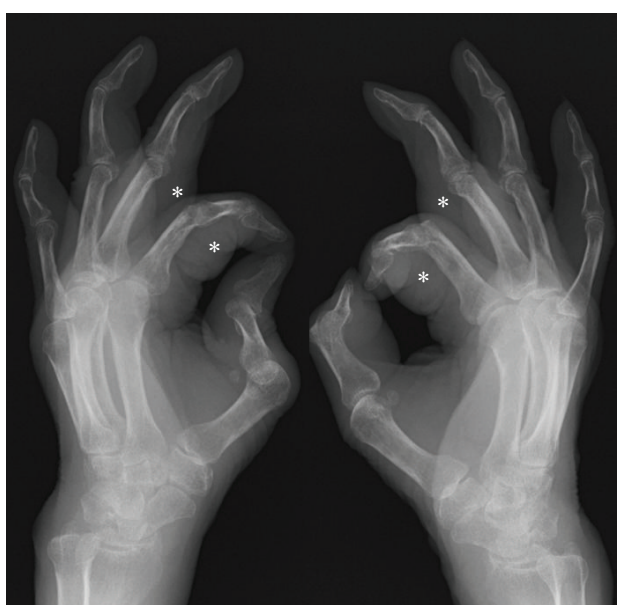

(b)

FIGURE 3: Plain radiographs of hands in Case 3. Plain radiographs of hands before treatment in the anterior-posterior view (a) and the oblique view (b). Arrows indicate small erosions in the heads of left 2 nd and 3rd metacarpal bones and right proximal phalanx. Markedly thickened soft tissues are demonstrated (asterisks).

intermittent fever, pancytopenia, multiple lymphadenopathy, and congestive heart failure. After receiving treatment for anemia and congestive heart failure with blood transfusion and diuretics, the patient underwent distal gastrectomy and proximal duodenectomy, on 28th of October, 2009 at the Department of Frontier Surgery, Chiba University Hospital, without any complication. The histopathological diagnosis was papillary adenocarcinoma.

On admission to our department, the patient had a low grade fever, lymphadenopathy in the neck and bilateral inguinal areas, and markedly swollen but only slightly tender fingers and wrists (Figures 2(a) and 2(b)). Blood tests 


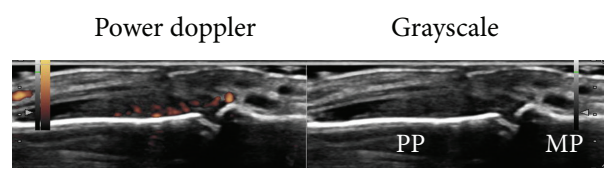

(a)

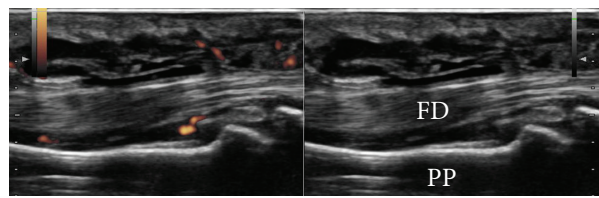

(b)

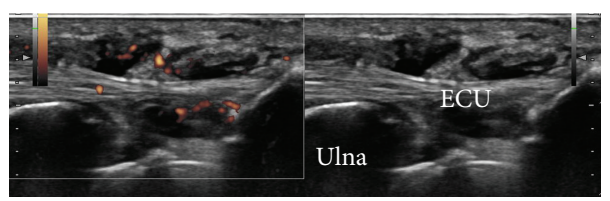

(c)

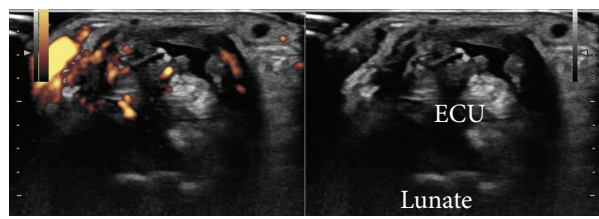

(d)

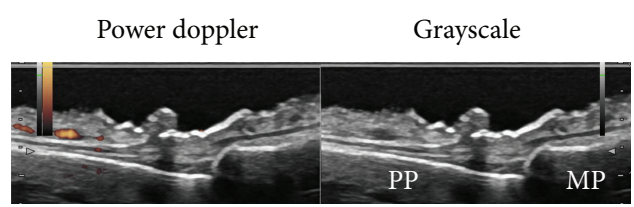

(e)

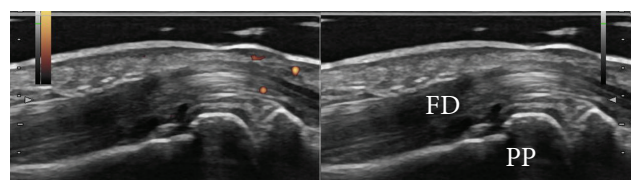

(f)

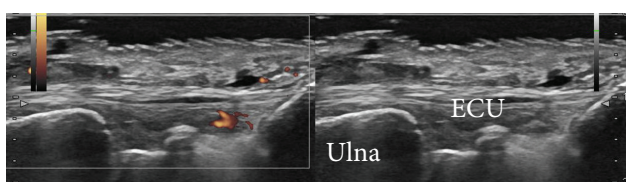

(g)

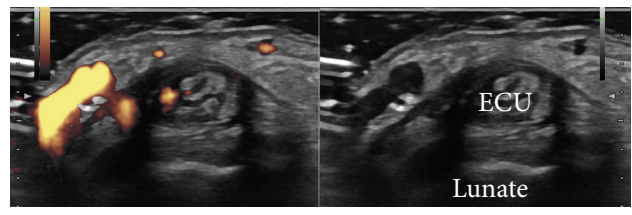

(h)

Figure 4: Ultrasound images of hands in Case 3 before and after treatment. Shown are the representative ultrasound images of the left hand before treatment $(\mathrm{a}-\mathrm{d})$ and after 24 weeks of abatacept treatment $(\mathrm{e}-\mathrm{h})$. Ultrasound images before treatment reveal moderate synovial hypertrophy with moderate Doppler signals in the 3rd proximal interphalangeal joint (a) and severe synovial hypertrophy with moderate Doppler signals in the flexor tendon of the 3rd finger (b) and extensor carpi ulnaris longitudinal view (c) (see also webvideo 1) and transverse view (d) (see also webvideo 2). Corresponding ultrasound images after abatacept treatment demonstrate marked improvement (e-h). PP: proximal phalanx; MP: middle phalanx; FD: flexor digitorum; ECU: extensor carpi ulnaris.

revealed bicytopenia (white blood cell count $1,900 / \mathrm{mm}^{3}$, hemoglobin $10.2 \mathrm{~g} / \mathrm{dL}$, platelet count $405 \times 10^{3} / \mathrm{mm}^{3}$ ), acute inflammatory response (CRP $32 \mathrm{mg} / \mathrm{L}$, erythrocyte sedimentation rate [ESR] $48 \mathrm{~mm} / \mathrm{h}$ ), hypergammaglobulinemia (IgG 2,395 mg/dL), abnormal coagulation (prothrombin time-international normalized ratio [PT-INR] 1.14, activated partial thromboplastin time [APTT] $40.1 \mathrm{sec}$, D-Dimer $23.2 \mu \mathrm{g} / \mathrm{mL}$ ), decreased levels of complements (C3 $29 \mathrm{mg} / \mathrm{dL}$, C4 $5 \mathrm{mg} / \mathrm{dL}, \mathrm{CH}_{50} 7 \mathrm{U} / \mathrm{mL}$ ), and the presence of autoantibodies (ANA x320 speckled pattern, IgG anti-DNA antibody $20.3 \mathrm{U} / \mathrm{mL}, \beta 2$-glycoprotein 1-dependent IgG anti-cardiolipin antibody $16 \mathrm{U} / \mathrm{mL}$, RF $188 \mathrm{U} / \mathrm{mL}$, and ACPA $>100 \mathrm{U} / \mathrm{mL}$ ). Antibodies to extractable nuclear antigens such as Sm, U1RNP, Ro/SS-A, and La/SS-B were all negative.

Hand radiographs showed soft tissue swelling, joint space narrowing, and bone erosions (Figure 3). Musculoskeletal ultrasound of the left hand revealed both intra-articular and tenosynovitis with increased PD signals (Figures 4(a)4(d), [see also webvideo 1, 2] see Supplementary Material available online at http://dx.doi.org/10.1155/2013/697525). Echocardiogram and computed tomography (CT) scan of chest showed a small amount of pericardial effusion. CT scan of abdomen and magnetic resonance imaging of brain were unremarkable.
The patient fulfilled both the 2010 ACR/EULAR Criteria for the Classification of RA (arthritis in more than ten joints, elevated levels of ESR and CRP, highly positive RF and ACPA, and disease duration of longer than 6 months) and the 1997 ACR Revised Classification Criteria of SLE (Pericarditis, leukocytopenia/lymphocytopenia, anti-DNA antibody/antiphospholipid antibody, and anti-nuclear antibody). Because she did not have severe organ involvement due to SLE, the treatment for RA was initiated with a small dose of MTX ( $4 \mathrm{mg} /$ week) given the presence of bicytopenia. CDAI gradually decreased from 35 to 23 with an increase to $6 \mathrm{mg} /$ week of MTX and she was discharged although low grade fever, bicytopenia, and serological activity of lupus persisted (Figure 5).

A small dose of prednisolone $(10 \mathrm{mg} /$ day $)$ was added to the treatment in July 2010 because of a gradual worsening of arthritis, persistent fever, and increased levels of anti-DNA antibody. Although the lupus-like manifestations, including fever, bicytopenia, and increased levels of anti-DNA antibody, improved after corticosteroid therapy; severe joint swelling persisted even after tacrolimus was added to the regimen and MTX was increased to $10 \mathrm{mg} /$ week.

Due to insufficient effectiveness, along with concerns regarding elevated liver enzymes, MTX was replaced 


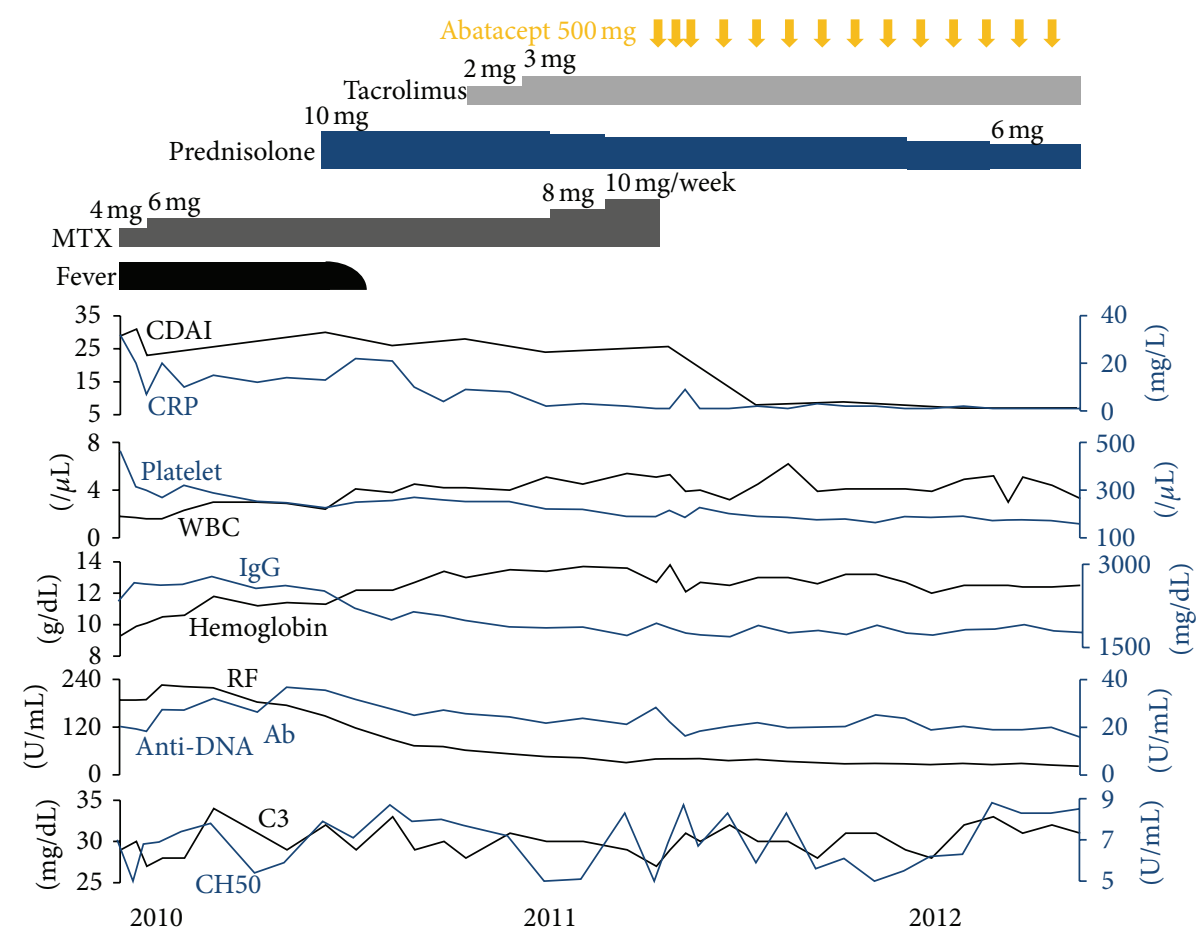

FIGURE 5: Treatment summary and clinical course of Case 3. MTX: methotrexate; CDAI: Clinical Disease Activity Index; CRP: C-reactive protein; WBC: white blood cell count; IgG: immunoglobulin G; RF: rheumatoid factor; Anti-DNA Ab: anti-DNA antibody; C3: complement component 3; CH50: 50\% hemolytic complement activity of serum.1. Panush et al. [1].

by abatacept, in April 2011. Joint swelling markedly improved (Figures 2(c) and 2(d)) and the patient achieved CDAI/SDAI/DAS28 remission after 12 weeks of abatacept treatment (Figure 1, Case 3). Intra-articular and tenosynovitis on ultrasound also improved markedly during the 24 weeks of abatacept treatment (Figures 4(e)-4(h)). Corticosteroid dose was reduced successfully to $6 \mathrm{mg} /$ day in February 2012 and the patient has remained in remission for 14 months.

\section{Discussion}

This is the first report of rhupus patients treated with abatacept for arthritis. All rhupus patients whose arthritis was refractory to MTX and other antirheumatic agents such as TNF antagonists and calcineurin inhibitors achieved a moderate or better response after receiving abatacept treatment. Although this result cannot be directly compared with data from clinical trials, the efficacy of abatacept for arthritis in rhupus patients seems to be at least as good as that in MTX-resistant and biologics-naive RA patients $[12,15]$. Considering the potentially detrimental effect of TNF antagonists on lupus manifestation, our data support the preferential choice of abatacept in a patient with rhupus syndrome whose arthritis is refractory to MTX. Whether this also applies to RA patients with positive ANA or anti-DNA antibody without clinical lupus manifestation is a matter of interest, and a comparison between different agents in this subpopulation is needed to address this question.
The relatively safe profile of abatacept as compared to other biological agents for infection in RA patients has been shown in clinical trials and a meta-analysis [12, 20]. Although the bursitis which Case 5 developed in this study could have been due to infection, this event required neither hospitalization nor intravenous administration of antibiotics and did not recur after readministration of abatacept. We think this nonserious adverse event, which could have been infectious, does not necessarily raise a concern about the relative safety of abatacept as compared to other biological agents, but further investigation is nonetheless needed for rhupus patients.

Ultrasound revealed active intra-articular synovitis in all cases and active tenosynovitis in the majority of our cases. The latter lesions may represent the pathology characteristic to SLE rather than RA according to the previous studies which showed high prevalence of tenosynovitis in lupus patients $[4,5,21]$. Although the absence of tenosynovitis did not influence the efficacy of abatacept in our small number of rhupus patients, the discrimination between intracapsular- and extra-capsular-dominant patients using ultrasound may be informative in the prediction of effectiveness of antirheumatic and immunosuppressive agents for arthritis in RA and SLE. A large-scale prospective study, however, would be necessary to prove this hypothesis.

In contrast to the efficacy of abatacept on arthritis, its efficacy on non-articular lupus manifestations was marginal in our case series, which is consistent with the previous study 
in patients with non-life-threatening SLE [19]. However, the statistically significant decrease in the levels of IgG and anti-DNA antibody in our cases may reflect the effect of abatacept on autoantibody production in SLE. Because T cellAPC interaction is an attractive target in the pathogenesis of SLE [22-25], more severe cases may respond to abatacept treatment. Accumulation of such cases may justify future trials to identify the subset of SLE patients who may benefit from abatacept treatment.

\section{Conclusions}

Treatment with abatacept is likely to be efficacious in patients with rhupus whose arthritis is refractory to methotrexate. In addition, abatacept may have a moderate effect on abnormal antibody production in rhupus patients.

\section{Conflict of Interests}

The authors declare that there is no conflict of interests regarding the publication of this paper.

\section{References}

[1] R. S. Panush, L. Edwards, S. Longley, and E. Webster, "Rhupus' syndrome," Archives of Internal Medicine, vol. 148, no. 7, pp. 1633-1636, 1988.

[2] R. M. van Vugt, R. H. Derksen, L. Kater, and J. W. J. Bijlsma, "Deforming arthropathy or lupus and rhupus hands in systemic lupus erythematosus," Annals of the Rheumatic Diseases, vol. 57, no. 9, pp. 540-544, 1998.

[3] M. T. Chan, P. Owen, J. Dunphy et al., "Associations of erosive arthritis with anti-cyclic citrullinated peptide antibodies and MHC class II alleles in systemic lupus erythematosus," Journal of Rheumatology, vol. 35, no. 1, pp. 77-83, 2008.

[4] E. M. Ball and A. L. Bell, "Lupus arthritis-do we have a clinically useful classification?" Rheumatology, vol. 51, no. 5, Article ID ker381, pp. 771-779, 2012.

[5] A. Gabba, M. Piga, A. Vacca et al., "Joint and tendon involvement in systemic lupus erythematosus: an ultrasound study of hands and wrists in 108 patients," Rheumatology, vol. 51, no. 12, pp. 2278-2285, 2012.

[6] C. Eriksson, S. Engstrand, K.-G. Sundqvist, and S. RantapääDahlqvist, "Autoantibody formation in patients with rheumatoid arthritis treated with anti-TNF $\alpha$," Annals of the Rheumatic Diseases, vol. 64, no. 3, pp. 403-407, 2005.

[7] P. J. Charles, R. J. Smeenk, J. De Jong et al., "Assessment of antibodies to double-stranded DNA induced in rheumatoid arthritis patients following treatment with infliximab, a monoclonal antibody to tumor necrosis factor alpha: findings in open-label and randomized placebo-controlled trials," Arthritis and Rheumatism, vol. 43, no. 11, pp. 2383-2390, 2000.

[8] M. F. Costa, N. R. Said, and B. Zimmermann, "Drug-induced lupus due to anti-tumor necrosis factor alpha agents," Seminars in Arthritis and Rheumatism, vol. 37, no. 6, pp. 381-387, 2008.

[9] M. De Bandt, J. Sibilia, X. Le Loët et al., "Systemic lupus erythematosus induced by anti-tumour necrosis factor alpha therapy: a French national survey," Arthritis Research \& Therapy, vol. 7, no. 3, pp. R545-R551, 2005.
[10] M. Debandt, O. Vittecoq, V. Descamps, X. Le Loët, and O. Meyer, "Anti-TNF- $\alpha$-induced systemic lupus syndrome," Clinical Rheumatology, vol. 22, no. 1, pp. 56-61, 2003.

[11] A. R. Chogle, C. V. Shah, and A. K. Murthy, "Role of anti-tumor necrosis factor- $\alpha$ blockers in inducing lupus erythematosus tumidus in 'rhupus syndrome,' Journal of Rheumatology, vol. 38, no. 6, pp. 1218-1219, 2011.

[12] M. Schiff, M. Keiserman, C. Codding et al., "Efficacy and safety of abatacept or infliximab vs placebo in ATTEST: a phase III, multi-centre, randomised, double-blind, placebocontrolled study in patients with rheumatoid arthritis and an inadequate response to methotrexate," Annals of the Rheumatic Diseases, vol. 67, no. 8, pp. 1096-1103, 2008.

[13] R. Westhovens, M. Robles, A. C. Ximenes et al., "Clinical efficacy and safety of abatacept in methotrexate-naive patients with early rheumatoid arthritis and poor prognostic factors," Annals of the Rheumatic Diseases, vol. 68, no. 12, pp. 1870-1877, 2009.

[14] M. C. Genovese, J. Becker, M. Schiff et al., "Abatacept for rheumatoid arthritis refractory to tumor necrosis factor $\alpha$ inhibition," New England Journal of Medicine, vol. 353, no. 11, pp. 1114-1123, 2005.

[15] J. M. Kremer, H. K. Genant, L. W. Moreland et al., "Effects of abatacept in patients with methotrexate-resistant active rheumatoid arthritis: a randomized trial," Annals of Internal Medicine, vol. 144, no. 12, pp. 865-876, 2006.

[16] R. Westhovens, J. M. Kremer, L. W. Moreland et al., "Safety and efficacy of the selective costimulation modulator abatacept in patients with rheumatoid arthritis receiving background methotrexate: a 5-year extended phase IIB study," Journal of Rheumatology, vol. 36, no. 4, pp. 736-742, 2009.

[17] F. K. Lekpa, V. Farrenq, F. Canouï-Poitrine et al., "Lack of efficacy of abatacept in axial spondylarthropathies refractory to tumor-necrosis-factor inhibition," Joint Bone Spine, vol. 79, no. 1, pp. 47-50, 2012.

[18] I. H. Song, F. Heldmann, M. Rudwaleit et al., "Treatment of active ankylosing spondylitis with abatacept: an open-label, 24week pilot study," Annals of the Rheumatic Diseases, vol. 70, no. 6, pp. 1108-1110, 2011.

[19] J. T. Merrill, R. Burgos-Vargas, R. Westhovens et al., "The efficacy and safety of abatacept in patients with non-lifethreatening manifestations of systemic lupus erythematosus: results of a twelve-month, multicenter, exploratory, phase IIb, randomized, double-blind, placebo-controlled trial," Arthritis and Rheumatism, vol. 62, no. 10, pp. 3077-3087, 2010.

[20] J. A. Singh, G. A. Wells, R. Christensen et al., "Adverse effects of biologics: a network meta-analysis and Cochrane overview," Cochrane Database of Systematic Reviews, vol. 2, Article ID CD008794, 2011.

[21] A. Delle Sedie, L. Riente, C. A. Scirè et al., "Ultrasound imaging for the rheumatologist XXIV. Sonographic evaluation of wrist and hand joint and tendon involvement in systemic lupus erythematosus," Clinical and Experimental Rheumatology, vol. 27, no. 6, pp. 897-901, 2009.

[22] G. C. Tsokos, "Mechanisms of disease: systemic lupus erythematosus," New England Journal of Medicine, vol. 365, no. 22, pp. 2110-2121, 2011.

[23] Y. H. Lee, J. B. Harley, and S. K. Nath, "CTLA-4 polymorphisms and systemic lupus erythematosus (SLE): a meta-analysis," Human Genetics, vol. 116, no. 5, pp. 361-367, 2005. 
[24] T. Takeuchi, K. Suzuki, T. Kondo, K. Yoshimoto, and K. Tsuzaka, "CD3 zeta defects in systemic lupus erythematosus," Annals of the Rheumatic Diseases, vol. 71, supplement 2, pp. i78-i81, 2012.

[25] T. Dörner, C. Giesecke, and P. E. Lipsky, "Mechanisms of B cell autoimmunity in SLE," Arthritis Research and Therapy, vol. 13, no. 5 , p. $243,2011$. 


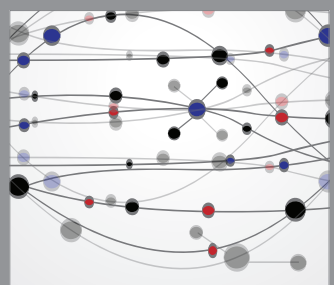

The Scientific World Journal
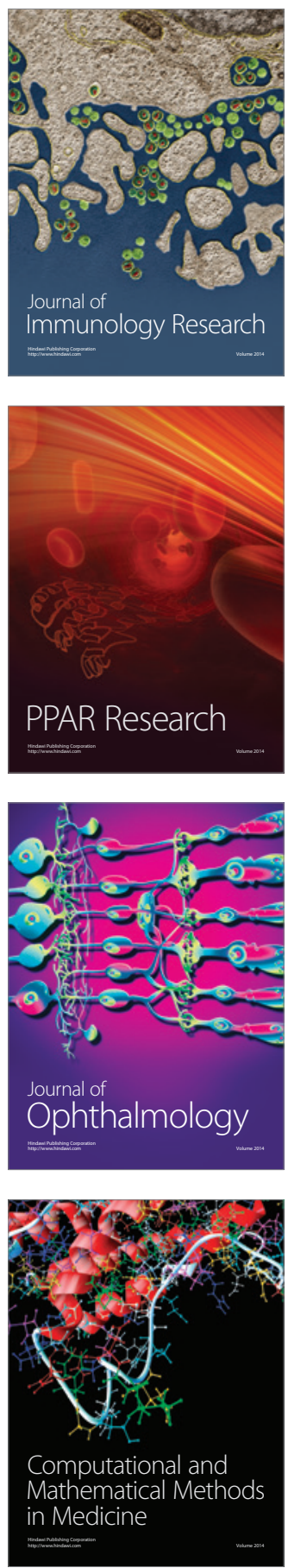

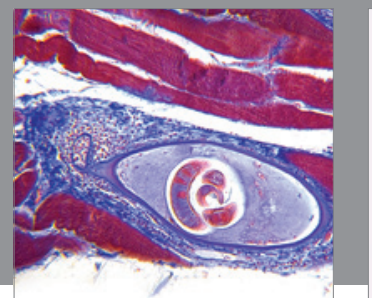

Gastroenterology

Research and Practice
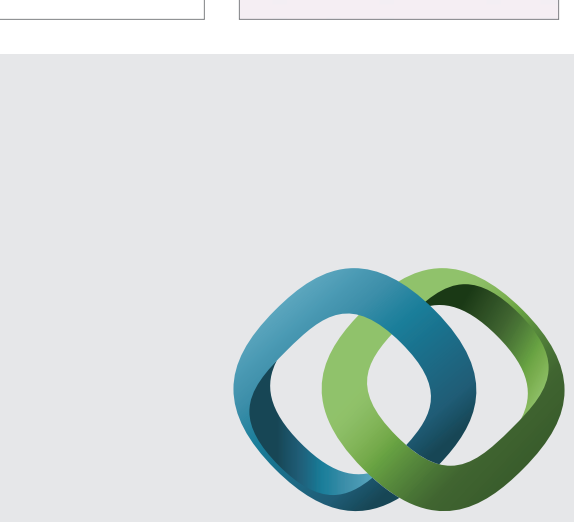

\section{Hindawi}

Submit your manuscripts at

http://www.hindawi.com
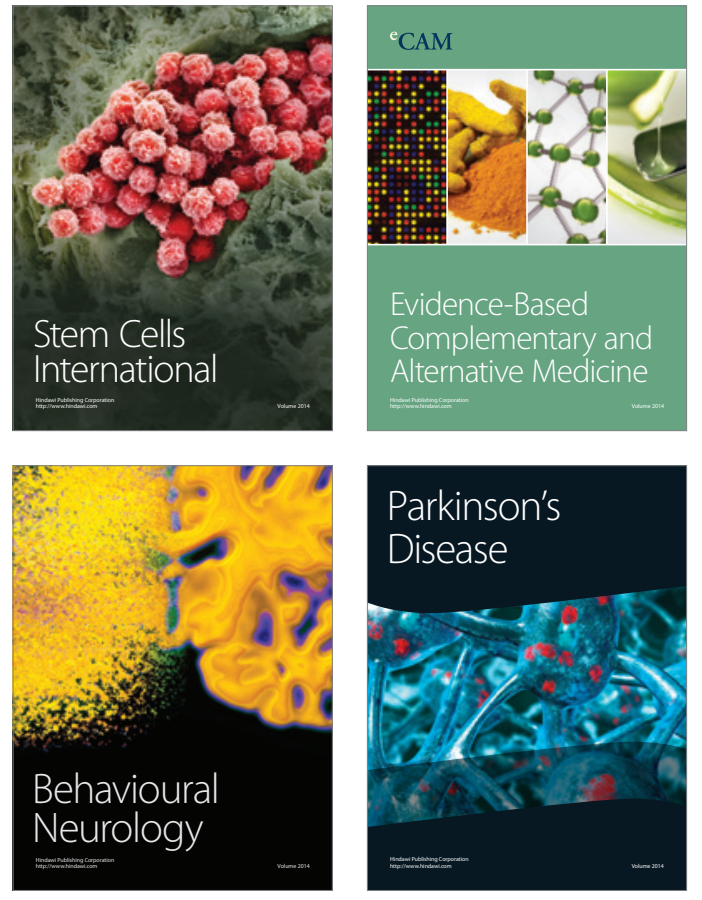
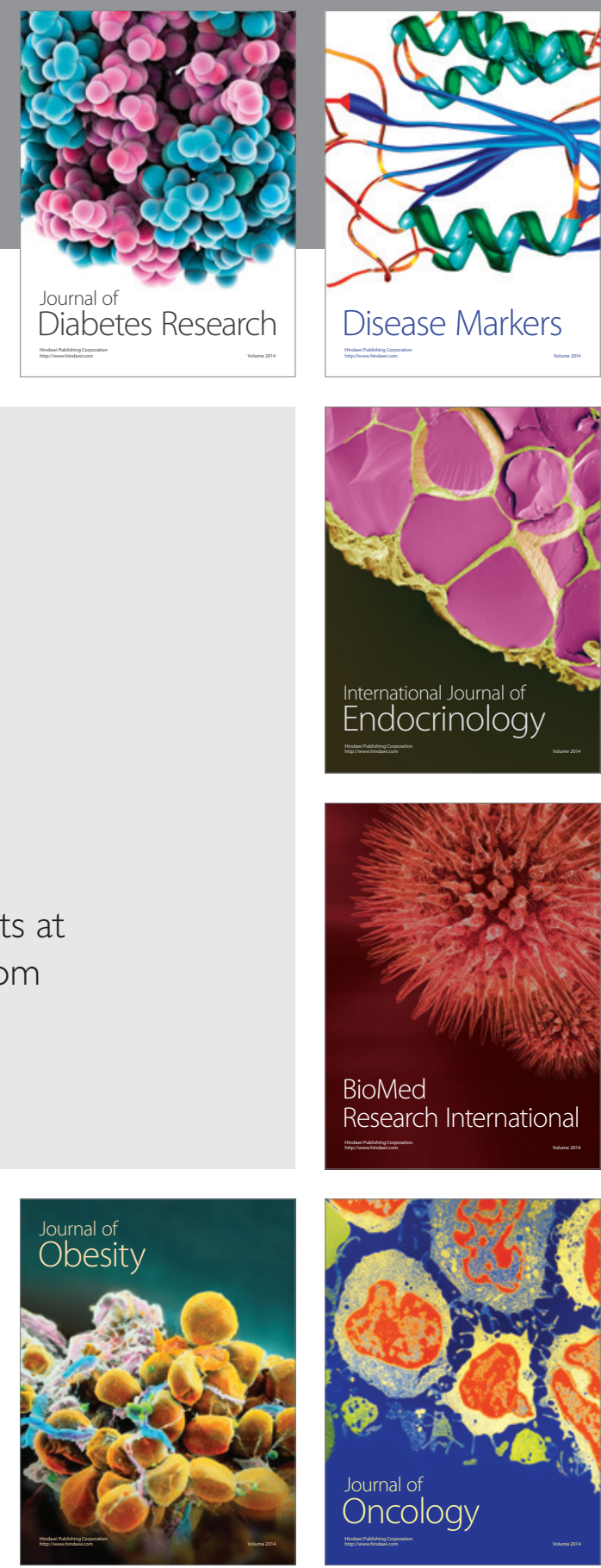

Disease Markers
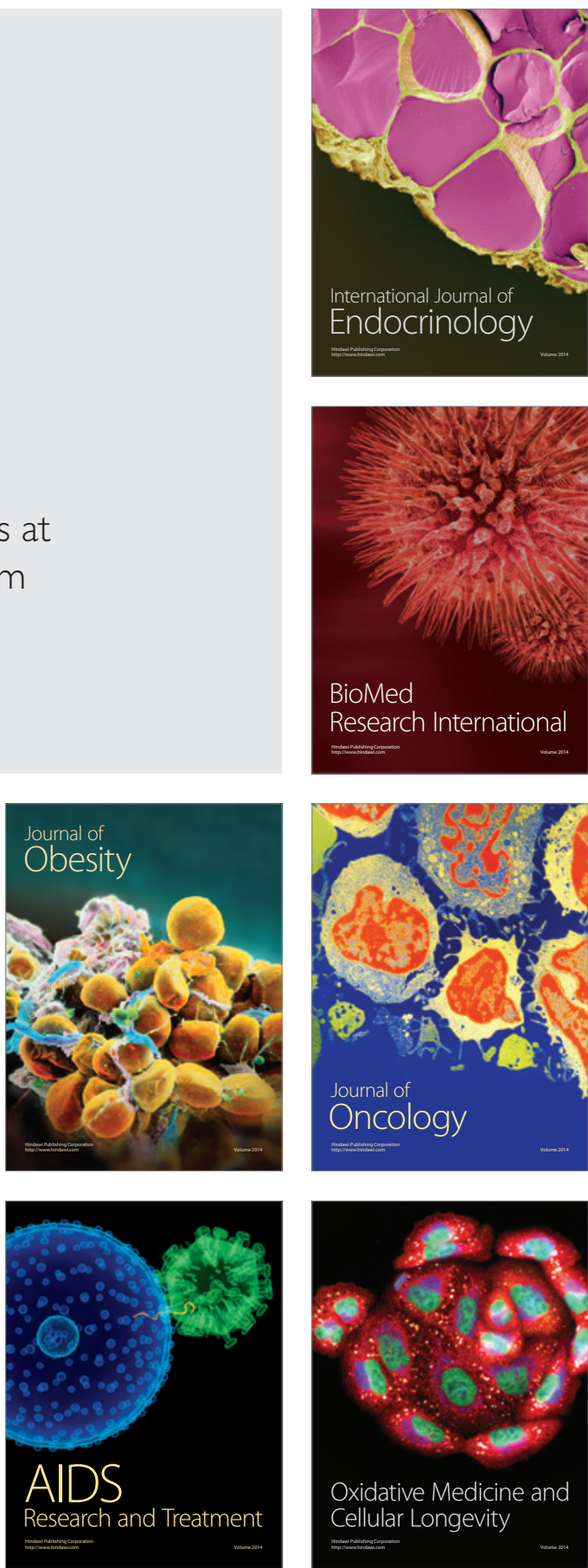